Kragujevac Journal of Mathematics

Volume 44(1) (2020), Pages 75-88.

\title{
CONSERVATION LAWS OF THE TIME-FRACTIONAL ZAKHAROV-KUZNETSOV-BURGERS EQUATION
}

\author{
AZADEH NADERIFARD ${ }^{1}$, S. REZA HEJAZI ${ }^{2}$, AND ELHAM DASTRANJ ${ }^{3}$
}

\begin{abstract}
An important application of Lie group theory of differential equations is applied to study conservation laws of time-fractional Zakharov-Kuznetsov-Burgers (ZKB) equation with Riemann-Liouville and Caputo derivatives. This analysis is based on a modified version of Noether's theorem provided by Ibragimov to construct the conserved vectors of the equation. This is done by non-linearly self-adjointness of the equation which will be stated via a formal Lagrangian in the sequel.
\end{abstract}

\section{INTRODUCTION}

Fractional order differential equations (FDEs) are important concepts in physic, mathematics and engineering. The theory of derivatives and integrals of fractional order illustrate the previous time history in the mathematical models of natural phenomena.

In the recent years, FDEs have been widely used and have numerous applications in various fields of sciences, as example probability and statistics, engineering, chemistry, electro-chemistry, biology, economics, modeling, astrophysics, electronics, dynamics, thermodynamics, vibration, viscoelasticity, control theory, electromagnetic theory, signal processing, arheology, geology, polymer and systems identification [2, 6, 9-14, 16-19, 25-27, 29,30] and [37].

Conservation laws can be used in the analysis of the essential properties of the solutions, particularly, investigation of existence, uniqueness and stability of the solutions [22]. There are some methods for constructing of conservation laws for

Key words and phrases. Generalized Zakharov-Kuznetsov-Burgers equation, Riemann Liouviile derivative, Caputo fractional derivative, Lie point symmetry, fractional conservation laws.

2010 Mathematics Subject Classification. Primary: 34K37. Secondary: 76M60.

DOI $10.46793 / \mathrm{KgJMat} 2001.075 \mathrm{~N}$

Received: December 03, 2017.

Accepted: February 20, 2018. 
PDEs, for example the Noether's theorem [20] and Ibragimov's theorem [7]. Almost all of these methods can be used for differential equations with fractional derivatives.

Lukashchuk, considered the fractional generalizations of the Noether's operators without Lagrangian and derived conservation laws for an arbitrary time-fractional FPDEs by formal Lagrangian [15].

One of the most important PDE which has a vast application in solitary wave's theory is the ZKB equation, also it makes an important role in electromagnetic and describes the propagation of Langmuir waves in an ionized plasma. Some of its modified forms illustrate the interactions of small amplitude, high frequency waves with acoustic waves. There are useful articles for finding the solitary waves solutions (specially for ZKB equation), see $[5,32,34-36]$. In this article, we focus on the timefractional ZKB equation by omitting the details of derivation in the following form:

$$
\partial_{t}^{\alpha} u+a u u_{x}+b u_{x x x}+c u_{x y y}-d u_{x x}-e u_{y y}=0,
$$

where $\partial_{t}^{\alpha} u$ is the fractional derivative of order $\alpha$ and $\alpha(1<\alpha \leqslant 2)$ is the order of the time-fractional. Taking $\alpha=1$, Zakharov and Kuznetsov established non-linear evolution equation which is related to nonlinear ion-acoustic waves in magnetized plasma including cold ions and hot isothermal electrons. We can see some useful papers in the literature to study the applications of this equation, see $[33,38]$ for more details. This equation by omitting the details of derivatives can be written as

$$
u_{t}+a u u_{x}+b u_{x x x}+c u_{x y y}-d u_{x x}-e u_{y y}=0,
$$

where $a, b, c, d$ and $e$ are constant quantities which involve the physical quantities and $x, y, t$ are independent variables where $u(t, x, y)$ is the dependent variable indicates the wave profile. El-Bedwehy and Moslem acquired the ZKB equation from an electronpositron-ion plasma [1].

This paper is organized as follows. Section 2 describes some basic properties of timefractional derivatives and four particular cases of time-fractional of ZKB equation. In Section 3 Lie symmetry analysis of the fractional ZKB equation is investigated. In Section 4, the concept of non-linear self-adjointness of ZKB equation is studied and conservation laws of (1.1) are obtained by using the Noether's operators. Some conclusions are given in the last section.

\section{Notations of Time-Fractional Generalizations}

There are several types of definitions for fractional derivatives, such as RiemannLiouville derivative, Caputo derivative, the modified Riemann-Liouville derivative, Riesz derivative and etc. [28,31].

Functions that have no first-order derivative could have Riemann-Liouville derivative but could not have Caputo fractional derivative and on the other hand Caputo fractional derivative is related to physical models.

In this paper we adopt the fractional derivatives in Riemann-Liouville derivatives as $\mathcal{D}_{t}^{\alpha}$ and Caputo derivative as ${ }^{C} \mathcal{D}_{t}^{\alpha}$. 
Definition 2.1. Let $f(t) \in L^{1}(a, b)$, be the set of all integrable functions, the timefractional integrals and left-sided and right-sided time-fractional integrals of order $\alpha$ are defined respectively as follow:

$$
\begin{aligned}
J_{t}^{\alpha} f(t) & :=\frac{1}{\Gamma(\alpha)} \int_{a}^{t} f(\tau)(t-\tau)^{\alpha-1} d \tau \\
{ }_{0} J_{t}^{\alpha} f(t) & :=\frac{1}{\Gamma(\alpha)} \int_{0}^{t} f(\tau)(t-\tau)^{\alpha-1} d \tau \\
{ }_{t} J_{T}^{\alpha} f(t) & :=\frac{1}{\Gamma(\alpha)} \int_{t}^{T} f(\tau)(\tau-t)^{\alpha-1} d \tau
\end{aligned}
$$

where $t>0$ and $J_{t}^{0} f(t)=f(t)$.

Definition 2.2. For $\alpha>0$, the Riemann-Liouville time-fractional is defined as

$$
\mathcal{D}_{t}^{\alpha} f(t, x)= \begin{cases}\frac{1}{\Gamma(n-\alpha)} \frac{\partial^{n}}{\partial t^{n}} \int_{0}^{t} \frac{f(\xi, x)}{(t-\xi)^{\alpha+1-n}} d \xi, & n-1<\alpha<n, \\ \frac{d^{n}}{d t^{n}} f(t), & \alpha=n \in \mathbb{N} .\end{cases}
$$

Definition 2.3. The Caputo derivative of order $\alpha$ is defined as

$$
{ }^{C} \mathcal{D}_{t}^{\alpha} f(t, x)= \begin{cases}\frac{1}{\Gamma(n-\alpha)} \int_{0}^{t} \frac{1}{(t-\xi)^{\alpha+1-n}} \frac{\partial^{n} f(\xi, x)}{\partial \xi^{n}} d \xi, & n-1<\alpha<n, \\ \frac{d^{n}}{d t^{n}} f(t), & \alpha=n \in \mathbb{N} .\end{cases}
$$

Now we should introduce some notations. Let

$$
\begin{aligned}
& J_{t}^{\alpha}(\lambda f(t)+g(t))=\lambda J_{t}^{\alpha} f(t)+J_{t}^{\alpha} g(t), \\
& J_{t}^{\alpha}\left(J_{t}^{\beta} f(t)\right)=J_{t}^{\beta}\left(J_{t}^{\alpha} f(t)\right)=J_{t}^{\alpha+\beta} f(t), \\
& \mathcal{D}_{t}^{\alpha} f(t)=\mathcal{D}_{t}^{n}\left(J_{t}^{n-\alpha} f(t)\right)=\mathcal{D}_{t}^{n}\left(D_{t}^{-(n-\alpha)} f(t)\right), \\
& \mathcal{D}_{t}^{\alpha}\left(\mathcal{D}_{t}^{-\alpha} f(t)\right)=f(t), \\
& { }^{C} \mathcal{D}_{t}^{\alpha} c=0, \quad c \text { is constant, } \\
& { }^{C} \mathcal{D}_{t}^{\alpha} \mathcal{D}_{t}^{-\alpha} f(t)=f(t),
\end{aligned}
$$

where $\alpha \in \mathbb{R}$ such that $n-1<\alpha<n$ and $n \in \mathbb{N}$. The classical ZKB equation can be written as follows: $u_{t}=C[u]$,

$$
C[u]=-a u u_{x}-b u_{x x x}-c u_{x y y}+d u_{x x}+e u_{y y} .
$$


In this paper we consider four forms of time-fractional generalization of ZKB equation as

$$
\begin{aligned}
& u_{t}=J_{t}^{\alpha} C[u], \\
& u_{t}=\mathcal{D}_{t}^{1-\alpha} C[u], \\
& u_{t}=C\left[J_{t}^{\alpha} f\right], \\
& u_{t}=C\left[\mathcal{D}_{t}^{1-\alpha} u\right],
\end{aligned}
$$

where $\mathcal{D}_{t}^{1-\alpha}$ and $J_{t}^{\alpha}$ are left-sided fractional Riemann-Liouville derivative of order $1-\alpha$ and Riemann-Liouville integral of order $\alpha$, respectively.

One can rewrite (2.3)-(2.6), so that their right-hand sides are exactly the right-hand side of (1.1). For this, we act on each of (2.3)-(2.6) by different operators.

Now, by acting the operator $\mathcal{D}_{t}^{\alpha}$ on (2.3) and denoting the dependent variable $u$ by $v$, and using formula (2.2), we can rewrite (2.3) as:

$$
\mathcal{D}_{t}^{\alpha} v_{t}=-a v v_{x}-b v_{x x x}-c v_{x y y}+d v_{x x}+e v_{y y} .
$$

By actting classical integral operator on (2.4) with respect to $t$, we have

$$
u(t, x)-u(0, x)=J_{t}^{\alpha}\left(-a u u_{x}-b u_{x x x}-c u_{x y y}+d u_{x x}+e u_{y y}\right) .
$$

Now we act the operator ${ }^{C} D_{t}^{\alpha}$ on the above equation and denote the dependent variable $u$ by $v$. We get

$$
{ }^{C} \mathcal{D}_{t}^{\alpha} v=-a v v_{x}-b v_{x x x}-c v_{x y y}+d v_{x x}+e v_{y y} .
$$

In (2.5), we denote a new non-local dependent variable $v$ by $J_{t}^{\alpha} u$, then we have $u=\mathcal{D}_{t}^{\alpha} v$. Hence this equation can be rewritten as

$$
\mathcal{D}_{t}^{\alpha+1} v=-a v v_{x}-b v_{x x x}-c v_{x y y}+d v_{x x}+e v_{y y} .
$$

In (2.6), by taking $u=J_{t}^{1-\alpha} v$, we obtain $u_{x}=J_{t}^{1-\alpha} v_{x}$ and other expressions. Finally this equation can be rewritten as:

$$
\mathcal{D}_{t}^{\alpha} v=-a v v_{x}-b v_{x x x}-c v_{x y y}+d v_{x x}+e v_{y y} .
$$

Thus, four different time-fractional (2.7)-(2.10) are different forms of time-fractional generalization of ZKB equation. After replacing $v$ by $u$, we can formally rewrite the (2.7)-(2.10) as

$$
\begin{aligned}
\mathcal{D}_{t}^{\alpha} u_{t} & =-a u u_{x}-b u_{x x x}-c u_{x y y}+d u_{x x}+e u_{y y}, \\
{ }^{C} \mathcal{D}_{t}^{\alpha} u & =-a u u_{x}-b u_{x x x}-c u_{x y y}+d u_{x x}+e u_{y y}, \\
\mathcal{D}_{t}^{\alpha+1} u & =-a u u_{x}-b u_{x x x}-c u_{x y y}+d u_{x x}+e u_{y y}, \\
\mathcal{D}_{t}^{\alpha} u & =-a u u_{x}-b u_{x x x}-c u_{x y y}+d u_{x x}+e u_{y y} .
\end{aligned}
$$

Clearly these equations coincide with the classical ZKB (1.2) in the limiting case of $\alpha=1$. In this paper, the order of time-fractional differential, in all of equations 
belongs to $(1,2)$. So $1<\alpha<2$. By using of summary mode of (2.11)-(2.14), we have

$$
F\left(t, x, y, u, \mathcal{D}_{t}^{\mu(\alpha)} u, u_{x}, \ldots, u_{x y y}\right)=-a u u_{x}-b u_{x x x}-c u_{x y y}+d u_{x x}+e u_{y y},
$$

where $\mathcal{D}_{t}^{\mu(\alpha)}$ denotes Riemann-Liouville operator or Caputo operator in (2.11)-(2.14).

\section{Lie Symmetry Analysis of the Time-Fractional Generalized ZKB EQUATION}

In this paper we consider Lie symmetry method in order to find conservation laws of the ZKB equation $[4,22,23,39]$. Consider one-parameter Lie group of infinitesimal transformations for that $(2.15)$

$$
\begin{aligned}
\bar{t} & =t+\varepsilon \tau(t, x, y, u)+O\left(\varepsilon^{2}\right), \\
\bar{x} & =x+\varepsilon \xi(t, x, y, u)+O\left(\varepsilon^{2}\right), \\
\bar{y} & =y+\varepsilon \rho(t, x, y, u)+O\left(\varepsilon^{2}\right), \\
\bar{u} & =u+\varepsilon \eta(t, x, y, u)+O\left(\varepsilon^{2}\right), \\
\bar{u}_{\bar{t}}^{\alpha} & =u_{t}^{\alpha}+\varepsilon \eta_{t}^{\alpha}(t, x, y, u)+O\left(\varepsilon^{2}\right), \\
\bar{u}_{\bar{x}} & =u_{x}+\varepsilon \eta^{x}(t, x, y, u)+O\left(\varepsilon^{2}\right), \\
\bar{u}_{\bar{x} \bar{x}} & =u_{x x}+\varepsilon \eta^{x x}(t, x, y, u)+O\left(\varepsilon^{2}\right), \\
\bar{u}_{\bar{x} \bar{x} \bar{x}} & =u_{x x x}+\varepsilon \eta^{x x x}(t, x, y, u)+O\left(\varepsilon^{2}\right), \\
\bar{u}_{\bar{y} \bar{y}} & =u_{y y}+\varepsilon \eta^{y y}(t, x, y, u)+O\left(\varepsilon^{2}\right), \\
\bar{u}_{\bar{y} \bar{y} \bar{x}} & =u_{y y x}+\varepsilon \eta^{y y x}(t, x, y, u)+O\left(\varepsilon^{2}\right),
\end{aligned}
$$

where $\varepsilon$ is the group parameter, then the associated Lie algebra of symmetries is the set of vector fields of the form

$$
X=\tau(t, x, y, u) \frac{\partial}{\partial t}+\xi(t, x, y, u) \frac{\partial}{\partial x}+\rho(t, x, y, u) \frac{\partial}{\partial y}+\eta(t, x, y, u) \frac{\partial}{\partial u},
$$

where

$$
\begin{aligned}
& \left.\frac{d \bar{t}}{d \varepsilon}\right|_{\varepsilon=0}=\tau(t, x, y, u),\left.\quad \frac{d \bar{x}}{d \varepsilon}\right|_{\varepsilon=0}=\xi(t, x, y, u), \\
& \left.\frac{d \bar{y}}{d \varepsilon}\right|_{\varepsilon=0}=\rho(t, x, y, u),\left.\quad \frac{d \bar{u}}{d \varepsilon}\right|_{\varepsilon=0}=\eta(t, x, y, u) .
\end{aligned}
$$

The third order prolongation of (3.2) leaves invariant (2.15). In other words

$$
\left.X^{(\alpha, 3)}\left(F\left(t, x, y, u, \mathcal{D}_{t}^{\mu(\alpha)} u, u_{x}, \ldots, u_{x y y}\right)\right)\right|_{(F=0)}=0,
$$

satisfied on solutions of (2.15), where $X^{(\alpha, 3)}$ is the third prolongation of the generator (3.2). By keeping the essential terms we have

$$
X^{(\alpha, 3)}=X+\eta^{x} \frac{\partial}{\partial u_{x}}+\eta^{x x} \frac{\partial}{\partial u_{x x}}+\eta^{x x x} \frac{\partial}{\partial u_{x x x}}+\eta^{y y} \frac{\partial}{\partial u_{y y}}+\eta^{y y x} \frac{\partial}{\partial u_{y y x}}+\eta_{t}^{\alpha} \frac{\partial}{\partial u_{t}^{\alpha}}
$$


Expanding the invariance condition (3.3) yields

$$
\eta_{t}^{\alpha}+a \eta u_{x}+a u \eta^{x}+b \eta^{x x x}+c \eta^{y y x}-d \eta^{x x}-e \eta^{y y}=0 .
$$

The prolongation coefficients are

$$
\begin{aligned}
\eta_{t}^{\alpha} & =\mathcal{D}_{t}^{\alpha}\left(\eta-\tau u_{t}-\xi u_{x}-\rho u_{y}\right)+\tau \mathcal{D}_{t}^{\alpha}\left(u_{t}\right)+\xi \mathcal{D}_{t}^{\alpha}\left(u_{x}\right)+\rho \mathcal{D}_{t}^{\alpha}\left(u_{y}\right), \\
\eta^{x} & =D_{x}\left(\eta-\tau u_{t}-\xi u_{x}-\rho u_{y}\right)+\tau u_{t x}+\xi u_{x x}+\rho u_{y x}, \\
\eta^{x x} & =D_{x x}\left(\eta-\tau u_{t}-\xi u_{x}-\rho u_{y}\right)+\tau u_{t x x}+\xi u_{x x x}+\rho u_{y x x}, \\
\eta^{x x x} & =D_{x x x}\left(\eta-\tau u_{t}-\xi u_{x}-\rho u_{y}\right)+\tau u_{t x x x}+\xi u_{x x x x}+\rho u_{y x x x}, \\
\eta^{y y} & =D_{y y}\left(\eta-\tau u_{t}-\xi u_{x}-\rho u_{y}\right)+\tau u_{t y y}+\xi u_{x y y}+\rho u_{y y y}, \\
\eta^{x y y} & =D_{y y x}\left(\eta-\tau u_{t}-\xi u_{x}-\rho u_{y}\right)+\tau u_{t y y x}+\xi u_{y y x x}+\rho u_{y y y x} .
\end{aligned}
$$

By using the generalized chain rule for a composite function and the generalized Leibnitz rule, we have the explicit form of $\eta_{t}^{\alpha}$ (see [18,21,24]),

$$
\begin{aligned}
\eta_{t}^{\alpha}= & \sum_{n=1}^{\infty}\left[\left(\begin{array}{l}
\alpha \\
n
\end{array}\right) \partial_{t}^{n} \eta_{u}-\left(\begin{array}{c}
\alpha \\
n+1
\end{array}\right) D_{t}^{n+1}(\tau)\right] \partial_{t}^{\alpha-n} u+\partial_{t}^{\alpha} \eta-u \partial_{t}^{\alpha} \eta_{u} \\
& -\sum_{n=1}^{\infty}\left(\begin{array}{l}
\alpha \\
n
\end{array}\right) D_{t}^{n}(\xi) \partial_{t}^{\alpha-n}\left(u_{x}\right)+\left(\eta_{u}-\alpha D_{t}(\tau)\right) \partial_{t}^{\alpha} u+\mu,
\end{aligned}
$$

where $\mu$ is

$$
\mu=\sum_{n=2}^{\infty} \sum_{m=2}^{n} \sum_{k=2}^{m} \sum_{l=0}^{k-1}\left(\begin{array}{l}
\alpha \\
n
\end{array}\right)\left(\begin{array}{l}
n \\
m
\end{array}\right)\left(\begin{array}{l}
k \\
l
\end{array}\right) \frac{t^{n-\alpha}}{k ! \Gamma(n+1-\alpha)}(-u)^{l} \frac{\partial^{m}}{\partial t^{m}}\left(u^{k-l}\right) \frac{\partial^{n-m+k} \eta}{\partial t^{n-m} \partial u^{k}} .
$$

After substituting the values of $\eta^{x}, \eta^{x x}, \eta^{x x x}, \eta^{y y}, \eta^{x y y}$ and $\eta_{t}^{\alpha}$ into (3.5) and equating the coefficients of derivatives $u$ to zero, the determining equations are obtained.

The solutions of this system are

$$
\xi=C_{1}, \quad \rho=C_{2}, \quad \tau=C_{3},
$$

where $C_{i}, i=1,2,3$, are arbitrary constants.

The lower limit of the integral in Riemann-Liouville derivative and Caputo derivative is fixed. So the condition $t=0$ should be invariant with respect to transformation (3.1) and therefore we have $\left.\tau(t, x, u)\right|_{t=0}=0$. So for $C_{3}=0$ vector field $\frac{\partial}{\partial t}$ is not a symmetry for (2.15).

Consequently, (2.15) admits two Lie point symmetries:

$$
X_{1}=\frac{\partial}{\partial x} \quad \text { and } \quad X_{2}=\frac{\partial}{\partial y} .
$$

\section{Conservation LaWs}

The theory of finding conservation laws for PDEs have a lot applications. This theory can describe some physically measures. Until three decades ago, all paper about conservation laws refer to problems with integer derivatives. 
Let us define components of a conservation law $C=\left(C^{t}, C^{x}, C^{y}\right)$ for $(2.15)$ in the same manner that is defined for PDEs. Let

$$
\begin{aligned}
C^{t} & =C^{t}(t, x, y, u, \ldots), \\
C^{x} & =C^{x}(t, x, y, u, \ldots), \\
C^{y} & =C^{y}(t, x, y, u, \ldots) .
\end{aligned}
$$

These components satisfy in

$$
\mathcal{D}_{t} C^{t}+\mathcal{D}_{x} C^{x}+\mathcal{D}_{y} C^{y}=0,
$$

on all solutions of (2.15).

Many definitions and concepts for constructing conservation laws of FDEs as the formal Lagrangian, the adjoint equation and Euler-Lagrangian operator are similar to PDEs. Emmy Noether illustrated symmetry and conservation law are connected for all linear and non-linear equations. Using Noether's theorem, the equation must be derived from the variational principle and have a Lagrangian in classical sense. Finding Lagrangian is not easy. In the other hand, there are equations that do not have classical Lagrangian.

In this paper, we construct the conservation laws of the ZKB fractional (2.15) via Ibragimove's method $[3,8]$.

The formal Lagrangian can be written

$$
\mathcal{L}=v F\left(t, x, y, u, \mathcal{D}_{t}^{\mu(\alpha)} u, u_{x}, \ldots, u_{x y y}\right),
$$

where $v$ is new dependent variable. We can define the formal Lagrangian for (2.15) by:

$$
\mathcal{L}=v \mathcal{D}_{t}^{\mu(\alpha)}+a v u u_{x}+b v u_{x x x}+c v u_{x y y}-d v u_{x x}-e v u_{y y}, \quad v=v(t, x, y) .
$$

The Euler-Lagrange operator with respect to $u$ for a finite time interval $t \in[0, T]$ is

$$
\frac{\delta}{\delta u}=\frac{\partial}{\partial u}+\left(\mathcal{D}_{t}^{\mu(\alpha)}\right)^{*} \frac{\partial}{\partial\left(\mathcal{D}_{t}^{\mu(\alpha)}\right)}+\sum_{m=1}^{\infty}(-1)^{m} D_{i_{1}} \cdots D_{i_{m}} \frac{\partial}{\partial u_{i_{1}, \ldots, i_{m}}},
$$

where $\left(\mathcal{D}_{t}^{\mu(\alpha)}\right)^{*}$ will be adjoint operator of $\left(\mathcal{D}_{t}^{\mu(\alpha)}\right)$. The adjoint operator is different for Riemann-Liouville derivative and Caputo fractional derivatives.

$\left(\mathcal{D}_{t}^{\alpha}\right)^{*}$ is adjoint operator for Riemman-Liouville derivative and $\left({ }^{C} \mathcal{D}_{t}^{\alpha}\right)^{*}$ is adjoint operator for Caputo fractional derivatives that are defined as follows (see [15])

$$
\begin{aligned}
& \left({ }_{0} \mathcal{D}_{t}^{\alpha}\right)^{*}=(-1)^{n}{ }_{t} J_{T}^{n-\alpha}\left(\mathcal{D}_{t}^{n}\right) \equiv{ }_{t}^{C} \mathcal{D}_{T}^{\alpha}, \\
& \left({ }_{0}^{C} \mathcal{D}_{t}^{\alpha}\right)^{*}=(-1)^{n} \mathcal{D}_{t}^{n}\left({ }_{t} I_{T}^{n-\alpha}\right) \equiv{ }_{t} \mathcal{D}_{T}^{\alpha},
\end{aligned}
$$

where ${ }_{t} J_{T}^{n-\alpha}$ is the right-sided fractional integral (2.1), ${ }_{t} \mathcal{D}_{T}^{\alpha}$ and ${ }_{t}^{C} \mathcal{D}_{T}^{\alpha}$ are the right-sided Riemann-Liouville and Caputo fractional derivative of order $\alpha$.

The adjoint operator $F^{*}$ of $(2.15)$ is

$$
F^{*}=\frac{\delta \mathcal{L}}{\delta u}=\left(\mathcal{D}_{t}^{\mu(\alpha)}\right)^{*} v-a v_{x} u-b v_{x x x}-c v_{x y y}-d v_{x x}-e v_{y y} .
$$


Then adjoint operator $\left(\mathcal{D}_{t}^{\mu(\alpha)}\right)^{*}$, for each of $(2.11)-(2.14)$ is

$$
\begin{array}{ll}
\text { for }(2.11) & \left(\mathcal{D}_{t}^{\mu(\alpha)}\right)^{*} \equiv\left(\mathcal{D}_{t}^{\alpha} \mathcal{D}_{t}\right)^{*}={ }_{t} \mathcal{D}_{T}^{\alpha} \mathcal{D}_{t}, \\
\text { for }(2.12) & \left(\mathcal{D}_{t}^{\mu(\alpha)}\right)^{*} \equiv\left({ }^{C} \mathcal{D}_{t}^{\alpha}\right)^{*}={ }_{t} \mathcal{D}_{T}^{\alpha}, \\
\text { for }(2.13) & \left(\mathcal{D}_{t}^{\mu(\alpha)}\right)^{*} \equiv\left(\mathcal{D}_{t}^{\alpha+1}\right)^{*}={ }_{t}^{C} \mathcal{D}_{T}^{\alpha+1}, \\
\text { for }(2.14) & \left(\mathcal{D}_{t}^{\mu(\alpha)}\right)^{*} \equiv\left(\mathcal{D}_{t}^{\alpha}\right)^{*}={ }_{t}^{C} \mathcal{D}_{T}^{\alpha} .
\end{array}
$$

Similar to PDEs, the fractional (2.15) is non-linearly self-adjoint, if there exists function $v=v(t, x, y)$ that solve the adjoint (4.3) for all solutions $u(x)$ of (2.15) and $v \neq 0[7]$.

Substituting $v=v(t, x, y)=\phi(t) \psi(x) \eta(y)$ into (4.3), yields:

$$
\begin{aligned}
& \psi^{\prime}(x) \eta(y) u+d \psi^{\prime \prime}(x) \eta(y)+b \psi^{\prime \prime \prime}(x) \eta(y)+e \psi(x) \eta^{\prime \prime}(y)+c \psi^{\prime}(x) \eta^{\prime \prime}(y)=0, \\
& \left(D_{t}^{\mu(\alpha)}\right)^{*}(\phi(t))=0 .
\end{aligned}
$$

The first equation in the above system is the second order PDE, which one of its solution is: $\psi(x) \eta(y)=\psi$, where $\psi \neq 0$ and is constant functions.

The second equation in system (4.8) depends on the type of fractional differential operator $\mathcal{D}_{t}^{\mu(\alpha)} u$, then (2.15) must be solved separately via each of equations (2.11)(2.14).

For (2.11) we have $\left(\mathcal{D}_{t}^{\alpha} \mathcal{D}_{t}(\Phi(t))\right)^{*}=0$, so by (4.4): $\Phi(t)=\phi_{1}(T-t)^{\alpha}+\phi_{2}$, for (2.12) we have $\left({ }^{C} \mathcal{D}_{t}^{\alpha}(\Phi(t))\right)^{*}=0$, so by (4.5): $\Phi(t)=\phi_{1}(T-t)^{\alpha}$, for Eq. (2.13) we have $\left(\mathcal{D}_{t}^{\alpha+1}(\Phi(t))\right)^{*}=0$, so by (4.6): $\Phi(t)=\phi_{1} t^{2}+\phi_{2} t+\phi_{3}$, for (2.14) we have $\left(\mathcal{D}_{t}^{\alpha}(\Phi(t))\right)^{*}=0$, so by (4.7): $\Phi(t)=\phi_{1} t+\phi_{2}$, where $\phi_{1}, \phi_{2}$ and $\phi_{3}$ are arbitrary constants. Note that for solving all of above equations we have used properties Riemann-Liouville and Caputo time-fractional derivatives.

In the Ibragimove's method, the components of conserved vector are obtained with effect the Noether's operators on the Lagrangian. Noether operators can be found from the fundamental operator identity, whose formula depends on the number of variables. The fundamental identity for ZKB equation with three independent variables $t, x, y$ and a dependent variable $u(t, x, y)$ can be written as follows:

$$
\bar{X}+\mathcal{D}_{t}(\tau) \mathcal{I}+\mathcal{D}_{x}(\xi) \mathcal{I}+\mathcal{D}_{y}(\rho) \mathcal{I}=W \frac{\delta}{\delta u}+\mathcal{D}_{t} \mathcal{N}^{t}+\mathcal{D}_{x} \mathcal{N}^{x}+\mathcal{D}_{y} \mathcal{N}^{y},
$$

where $\bar{X}$ is prolongation operator $(3.4), \mathcal{I}$ is identity operator, $\frac{\delta}{\delta u}$ is the EulerLagrangiane operator and $W$ is characteristic for Lie point group generator (3.2),

$$
W=\eta-\tau u_{t}-\xi u_{x}-\rho u_{y} .
$$

Finally $\mathcal{N}^{t}, \mathcal{N}^{x}$ and $\mathcal{N}^{y}$ are Noether operators. Because (2.15) do not have the fractional derivatives with respect to $x$ and $y$, definitions for them are exactly the 
same as general formula that are given for each of symmetries as follows (see [7]):

$$
\begin{aligned}
\mathcal{N}^{x}= & \xi \mathcal{I}+W\left(\frac{\partial}{\partial u_{x}}-\mathcal{D}_{i} \frac{\partial}{\partial u_{x i}}+\mathcal{D}_{i} \mathcal{D}_{k} \frac{\partial}{\partial u_{x i k}}-\cdots\right) \\
& +\mathcal{D}_{i}(W)\left(\frac{\partial}{\partial u_{x i}}-\mathcal{D}_{k} \frac{\partial}{\partial u_{x i k}}-\cdots\right)+\mathcal{D}_{i} \mathcal{D}_{k}(W)\left(\frac{\partial}{\partial u_{x i k}}-\ldots\right), \\
\mathcal{N}^{y}= & \rho \mathcal{I}+W\left(\frac{\partial}{\partial u_{y}}-\mathcal{D}_{i} \frac{\partial}{\partial u_{y i}}+\mathcal{D}_{i} \mathcal{D}_{k} \frac{\partial}{\partial u_{y i k}}-\cdots\right) \\
& +\mathcal{D}_{i}(W)\left(\frac{\partial}{\partial u_{y i}}-\mathcal{D}_{k} \frac{\partial}{\partial u_{y i k}}-\cdots\right)+\mathcal{D}_{i} \mathcal{D}_{k}(W)\left(\frac{\partial}{\partial u_{y i k}}-\cdots\right),
\end{aligned}
$$

where $i$ and $k$ are $x$ or $y$.

Since (2.15) has fractional derivatives respect to $t$, Nother's operator $\mathcal{N}^{t}$ for the case with the Riemann-Liouville time-fractional derivative is:

$$
\mathcal{N}^{t}=\tau \mathcal{I}+\sum_{k=0}^{n-1}(-1)^{k} \mathcal{D}_{t}^{\alpha-1-k}(W) \mathcal{D}_{t}^{k} \frac{\partial}{\partial\left(\mathcal{D}_{t}^{\alpha} u\right)}-(1)^{n} J\left(W, \mathcal{D}_{t}^{n} \frac{\partial}{\partial\left(\mathcal{D}_{t}^{\alpha} u\right)}\right)
$$

For the another case, with the Caputo time-fractional derivative $\mathcal{N}^{t}$ is

$$
\mathcal{N}^{t}=\tau \mathcal{I}+\sum_{k=0}^{n-1} \mathcal{D}_{t}^{k}(W) \mathcal{D}_{T}^{\alpha-1-k} \frac{\partial}{\partial\left(\mathcal{D}_{t}^{\alpha} u\right)}-J\left(\mathcal{D}_{t}^{n}(W), \frac{\partial}{\partial\left(\mathcal{D}_{t}^{\alpha} u\right)}\right) .
$$

In (4.12) and (4.13),

$$
J(f, g)=\frac{1}{\Gamma(n-\alpha)} \int_{0}^{t} \int_{t}^{T} \frac{f(\tau, x, y) g(\mu, x, y)}{(\mu-\tau)^{n-\alpha}} d \mu d \tau .
$$

This integral has the following property:

$$
\mathcal{D}_{t} J(f, g)=f_{t} \mathcal{J}_{T}^{n-\alpha} g-g_{0} \mathcal{J}_{t}^{n-\alpha} f .
$$

The (2.15) is non-linearly self-adjoint, because there exists a non-unique function $v=v(t, x, y)$ such that (4.3) is satisfied for any solution of (2.15). We act on both sides of (4.9) by formal Lagrangian (4.2). Because formal Lagrangian $\mathcal{L}$ vanishes on the solutions of (2.15), the left-hand side of equallity (4.9) is equal to zero:

$$
\begin{aligned}
\bar{X} \mathcal{L}+\mathcal{D}_{t}(\tau) \mathcal{I}(\mathcal{L})+\mathcal{D}_{x}(\xi) \mathcal{I}(\mathcal{L})+\mathcal{D}_{y}(\rho) \mathcal{I}(\mathcal{L}) & =\bar{X} \mathcal{L}+\mathcal{D}_{t}(\tau) \mathcal{L}+\mathcal{D}_{x}(\xi) \mathcal{L}+\mathcal{D}_{y}(\rho) \mathcal{L} \\
& =0,
\end{aligned}
$$

and by considering (4.9),

$$
W \frac{\delta \mathcal{L}}{\delta u}+\mathcal{D}_{t}\left(\mathcal{N}^{t} \mathcal{L}\right)+\mathcal{D}_{x}\left(\mathcal{N}^{x} \mathcal{L}\right)+\mathcal{D}_{y}\left(\mathcal{N}^{y} \mathcal{L}\right)=0
$$

Since for non-linearly self-adjoint equation this condition is valid, i.e., $\frac{\delta \mathcal{L}}{\delta u}=0$, so

$$
\mathcal{D}_{t}\left(\mathcal{N}^{t} \mathcal{L}\right)+\mathcal{D}_{x}\left(\mathcal{N}^{x} \mathcal{L}\right)+\left.\mathcal{D}_{y}\left(\mathcal{N}^{y} \mathcal{L}\right)\right|_{(2.15)}=0
$$


By comparing (4.1) and (4.14), we have

$$
C^{t}=\mathcal{N}^{t}(\mathcal{L}), \quad C^{x}=\mathcal{N}^{x}(\mathcal{L}), \quad C^{y}=\mathcal{N}^{y}(\mathcal{L}) .
$$

In the sequel, conserved vectors associated with different symmetries and different terms of (2.15) are constructed.

Now we will find the conservation laws of the (2.11). The formal Lagrangian for (2.11) after substitution acceptable $v$ is defined by

$$
\mathcal{L}=\left(\psi \phi_{1}(T-t)^{\alpha}+\psi \phi_{2}\right)\left(\mathcal{D}_{t}^{\mu(\alpha)}+a u u_{x}+b u_{x x x}+c u_{x y y}-d u_{x x}-e u_{y y}\right) .
$$

In this case, using (4.10), (4.11) and (4.13), one can get the components of conserved vectors:

$$
\begin{aligned}
C^{x}= & a\left(\psi \phi_{1}(T-t)^{\alpha}+\psi \phi_{2}\right) u W-d\left(\psi \phi_{1}(T-t)^{\alpha}+\psi \phi_{2}\right) W_{x} \\
& +b\left(\psi \phi_{1}(T-t)^{\alpha}+\psi \phi_{2}\right) W_{x x}+c\left(\psi \phi_{1}(T-t)^{\alpha}+\psi \phi_{2}\right) W_{y y}, \\
C^{y}= & e\left(\psi \phi_{1}(T-t)^{\alpha}+\psi \phi_{2}\right) W_{y}+c\left(\psi \phi_{1}(T-t)^{\alpha}+\psi \phi_{2}\right) W_{y y}, \\
C^{t}= & \mathcal{J}_{t}^{1-\alpha} W_{t} \psi \phi_{1}(T-t)^{\alpha}+\mathcal{J}_{t}^{1-\alpha} W_{t} \psi \phi_{2}+\mathcal{J}_{T}^{1-\alpha}\left(\alpha \psi \phi_{1}(T-t)^{\alpha-1}\right) W \\
& +J\left(W_{t}, \alpha \psi \phi_{1}(T-t)^{\alpha-1}\right) .
\end{aligned}
$$

By applying above equations and considering $W=-u_{x}$ coordinate with $X_{1}$ the following components are obtained:

$$
\begin{aligned}
C^{x}= & -a u_{x}\left(\psi \phi_{1}(T-t)^{\alpha}+\psi \phi_{2}\right) u+d u_{x x}\left(\psi \phi_{1}(T-t)^{\alpha}+\psi \phi_{2}\right) \\
& -b u_{x x}\left(\psi \phi_{1}(T-t)^{\alpha}+\psi \phi_{2}\right)-c u_{x y y}\left(\psi \phi_{1}(T-t)^{\alpha}+\psi \phi_{2}\right), \\
C^{y}= & -e u_{x y}\left(\psi \phi_{1}(T-t)^{\alpha}+\psi \phi_{2}\right)-c u_{x y y}\left(\psi \phi_{1}(T-t)^{\alpha}+\psi \phi_{2}\right), \\
C^{t}= & -\mathcal{J}_{t}^{1-\alpha} u_{x t} \psi \phi_{1}(T-t)^{\alpha}-\mathcal{J}_{t}^{1-\alpha} u_{x t} \psi \phi_{2}-u_{x} \Gamma(\alpha+1) \psi \phi_{1} \\
& +\alpha \psi \phi_{1} J\left(u_{x t},(T-t)^{\alpha-1}\right) .
\end{aligned}
$$

Similarly, by considering $X_{2}$, the conserved vectors are:

$$
\begin{aligned}
C^{x}= & -a u_{y}\left(\psi \phi_{1}(T-t)^{\alpha}+\psi \phi_{2}\right) u+d u_{x y}\left(\psi \phi_{1}(T-t)^{\alpha}+\psi \phi_{2}\right) \\
& -b u_{x x y}\left(\psi \phi_{1}(T-t)^{\alpha}+\psi \phi_{2}\right)-c u_{y y y}\left(\psi \phi_{1}(T-t)^{\alpha}+\psi \phi_{2}\right), \\
C^{y}= & -e u_{y y}\left(\psi \phi_{1}(T-t)^{\alpha}+\psi \phi_{2}\right)-c u_{y y y}\left(\psi \phi_{1}(T-t)^{\alpha}+\psi \phi_{2}\right), \\
C^{t}= & -u_{y t} \mathcal{J}_{t}^{1-\alpha} \psi \phi_{1}(T-t)^{\alpha}-\mathcal{J}_{t}^{1-\alpha} u_{y t} \psi \phi_{2}-\mathcal{J}_{T}^{1-\alpha}\left(\alpha \psi \phi_{1}(T-t)^{\alpha-1}\right) u_{y} \\
& -J\left(u_{t y}, \alpha \psi \phi_{1}(T-t)^{\alpha-1}\right) .
\end{aligned}
$$

The corresponding conserved vectors for (2.12)-(2.14) are presented in Tables 1 and 2 . 
TABle 1. Components of conservation laws for (2.12) and (2.13)

\begin{tabular}{|c|c|c|c|}
\hline$X_{i}$ & $W_{i}$ & $C^{x, y, t}$ & Components of conservation laws for (2.12) \\
\hline \multirow{3}{*}{$X_{1}$} & \multirow{3}{*}{$-u_{x}$} & $C^{x}$ & $\begin{array}{l}-a\left(\psi \phi_{1}(T-t)^{\alpha}\right) u_{x} u+d u_{x x}\left(\psi \phi_{1}(T-t)^{\alpha}\right) \\
-b u_{x x x}\left(\psi \phi_{1}(T-t)^{\alpha}\right)-c u_{x y y}\left(\psi \phi_{1}(T-t)^{\alpha}\right)\end{array}$ \\
\hline & & $C^{y}$ & $-e u_{x y}\left(\psi \phi_{1}(T-t)^{\alpha}\right)-c u_{x y y}\left(\psi \phi_{1}(T-t)^{\alpha}\right)$ \\
\hline & & $C^{t}$ & $-\phi_{1} \psi u_{x} \Gamma(\alpha)-\phi_{1} \psi u_{x t} \Gamma(\alpha)(T-t)+\phi_{1} \psi J\left(u_{x t t},(T-t)^{\alpha-1}\right)$ \\
\hline \multirow{3}{*}{$X_{2}$} & \multirow{3}{*}{$-u_{y}$} & $C^{x}$ & $\begin{array}{l}-a\left(\psi \phi_{1}(T-t)^{\alpha}\right) u_{y} u+d u_{x y}\left(\psi \phi_{1}(T-t)^{\alpha}\right) \\
-b u_{x x y}\left(\psi \phi_{1}(T-t)^{\alpha}\right)-c u_{y y y}\left(\psi \phi_{1}(T-t)^{\alpha}\right)\end{array}$ \\
\hline & & $C^{y}$ & $-e u_{y y}\left(\psi \phi_{1}(T-t)^{\alpha}\right)-c u_{y y y}\left(\psi \phi_{1}(T-t)^{\alpha}\right)$ \\
\hline & & $C^{t}$ & $-\phi_{1} \psi u_{y} \Gamma(\alpha)-\phi_{1} \psi u_{y t} \Gamma(\alpha)(T-t)+\phi_{1} \psi J\left(u_{y t t},(T-t)^{\alpha-1}\right)$ \\
\hline$X_{i}$ & $W_{i}$ & $C^{x, y, t}$ & Components of conservation laws for (2.13) \\
\hline \multirow{3}{*}{$X_{1}$} & \multirow{3}{*}{$-u_{x}$} & $C^{x}$ & $\begin{array}{l}-a\left(\psi \phi_{1} t^{2}+\psi \phi_{2} t+\psi \phi_{3}\right) u_{x} u+d u_{x x}\left(\psi \phi_{1} t^{2}+\psi \phi_{2} t+\psi \phi_{3}\right) \\
-b u_{x x x}\left(\psi \phi_{1} t^{2}+\psi \phi_{2} t+\psi \phi_{3}\right)-c u_{x y y}\left(\psi \phi_{1} t^{2}+\psi \phi_{2} t+\psi \phi_{3}\right)\end{array}$ \\
\hline & & $C^{y}$ & $-e u_{x y}\left(\psi \phi_{1} t^{2}+\psi \phi_{2} t+\psi \phi_{3}\right)-c u_{x y y}\left(\psi \phi_{1} t^{2}+\psi \phi_{2} t+\psi \phi_{3}\right)$ \\
\hline & & $C^{t}$ & $\begin{array}{l}\mathcal{D}_{t}^{\alpha}\left(-u_{x}\right)\left(\psi \phi_{1} t^{2}+\psi \phi_{2} t+\psi \phi_{3}\right)+\mathcal{D}_{t}^{\alpha-1}\left(u_{x}\right)\left(2 \psi \phi_{1} t+\psi \phi_{2}\right) \\
+J\left(u_{x}, 2 \psi \phi_{1}\right)\end{array}$ \\
\hline \multirow{3}{*}{$X_{2}$} & \multirow{3}{*}{$-u_{y}$} & $C^{x}$ & $\begin{array}{l}-a\left(\psi \phi_{1} t^{2}+\psi \phi_{2} t+\psi \phi_{3}\right) u_{y} u+d u_{x y}\left(\psi \phi_{1} t^{2}+\psi \phi_{2} t+\psi \phi_{3}\right) \\
-b u_{x x y}\left(\psi \phi_{1} t^{2}+\psi \phi_{2} t+\psi \phi_{3}\right)-c u_{y y y}\left(\psi \phi_{1} t^{2}+\psi \phi_{2} t+\psi \phi_{3}\right)\end{array}$ \\
\hline & & $C^{y}$ & $-e u_{y y}\left(\psi \phi_{1} t^{2}+\psi \phi_{2} t+\psi \phi_{3}\right)-c u_{y y y}\left(\psi \phi_{1} t^{2}+\psi \phi_{2} t+\psi \phi_{3}\right)$ \\
\hline & & $C^{t}$ & $\begin{array}{l}\mathcal{D}_{t}^{\alpha}\left(-u_{y}\right)\left(\psi \phi_{1} t^{2}+\psi \phi_{2} t+\psi \phi_{3}\right)+\mathcal{D}_{t}^{\alpha-1}\left(u_{y}\right)\left(2 \psi \phi_{1} t+\psi \phi_{2}\right) \\
+J\left(u_{y}, 2 \psi \phi_{1}\right)\end{array}$ \\
\hline
\end{tabular}


TABLE 2. Components of conservation laws for (2.14)

\begin{tabular}{|c|c|c|c|}
\hline$\overline{X_{i}}$ & $W_{i}$ & $C^{x, y, t}$ & Component of conservation laws for (2.14) \\
\hline \multirow{3}{*}{$X_{1}$} & \multirow{3}{*}{$-u_{x}$} & $C^{x}$ & $\begin{array}{l}-a\left(\psi \phi_{1} t+\psi \phi_{2}\right) u_{x} u+d u_{x x}\left(\psi \phi_{1} t+\psi \phi_{2}\right) \\
-b u_{x x x}\left(\psi \phi_{1} t+\psi \phi_{2}\right)-c u_{x y y}\left(\psi \phi_{1} t+\psi \phi_{2}\right)\end{array}$ \\
\hline & & $C^{y}$ & $-e u_{x y}\left(\psi \phi_{1} t+\psi \phi_{2}\right)-c u_{x y y}\left(\psi \phi_{1} t+\psi \phi_{2}\right)$ \\
\hline & & $C^{t}$ & $\mathcal{D}_{t}^{\alpha}\left(-u_{x}\right)\left(\psi \phi_{1} t+\psi \phi_{2}\right)+\mathcal{D}_{t}^{\alpha-2}\left(u_{x}\right) \phi_{1} \psi$ \\
\hline \multirow{3}{*}{$X_{2}$} & \multirow{3}{*}{$-u_{y}$} & $C^{x}$ & $\begin{array}{l}-a\left(\psi \phi_{1} t+\psi \phi_{2}\right) u_{y} u+d u_{x y}\left(\psi \phi_{1} t+\psi \phi_{2}\right) \\
-b u_{x x y}\left(\psi \phi_{1} t+\psi \phi_{2}\right)-c u_{y y y}\left(\psi \phi_{1} t+\psi \phi_{2}\right)\end{array}$ \\
\hline & & $C^{y}$ & $-e u_{y y}\left(\psi \phi_{1} t+\psi \phi_{2}\right)-c u_{y y y}\left(\psi \phi_{1} t+\psi \phi_{2}\right)$ \\
\hline & & $C^{t}$ & $\mathcal{D}_{t}^{\alpha}\left(-u_{y}\right)\left(\psi \phi_{1} t+\psi \phi_{2}\right)+\mathcal{D}_{t}^{\alpha-2}\left(u_{y}\right) \phi_{1} \psi$ \\
\hline
\end{tabular}

\section{Conclusion}

In this paper the time-fractional generalizations of the Zakharov-Kuznetsov-Burgers equation is studied. This is an important topic in investigation of nonlinear cold-ionacoustic waves and hot-isothermal electrons in magnetized plasma. The conservation laws of the equation is found via a modified version of Noether's theorem. This version is provided by Ibragimov and stated by considering a formal Lagrangian for a given PDE or FDE. Consequently, a generalized fractional version of Ibragomov's theorem between fractional symmetries and conservation laws are presented.

\section{REFERENCES}

[1] N. A. El-Bedwehyand and W. M. Moslem, Zakharov-Kuznetsov-Burgers equation in super thermal electron-positron-ion plasma, Astrophys. Space Sci. 335(2) (2011), 435-442.

[2] S. Das, Functional Fractional Calculus for System Identification and Controls, Springer-Verlag, Berlin, Heidelberg, 2008.

[3] R. K. Gazizov, N. H. Ibragimov and S. Y. Lukashchuk, Nonlinear self-adjointness, conservation laws and exact solutions of time-fractional Kompaneets equations, Commun. Nonlinear Sci. Numer. Simul. 23 (2014), 153-163.

[4] R. K. Gazizov, A. A. Kasatkin and S. Y. Lukashchuk, Group-invariant solutions of fractional differential equations, in: J. Machado, A. Luo, R. Barbosa, M. Silva, L. Figueiredo (Eds.), Nonlinear Science and Complexity, Springer, Dordrecht, 2011, 51-59.

[5] M. A. Helal and A. R. Seadawy, Benjamin-Feirin stability in nonlinear dispersive waves, Comput. Math. Appl. 64 (2012), 3557-3568.

[6] R. Hilfer, Applications of Fractional Calculus in Physics, World Scientific, River Edge, 2000.

[7] N. H. Ibragimov, Nonlinear self-adjointness in constructing conservation laws, Archives of ALGA 7/8 (2011), 1-99. 
[8] N.H Ibragimov, A new conservation theorem, J. Math. Anal. Appl. 333 (2007), 311-328.

[9] Y. L. Jiang and X. L. Ding, Non-negative solutions of fractional functional differential equations, Comput. Math. Appl. 5 (2012), 896-904.

[10] A. A. Kilbas, H. M. Srivastava and J. J. Trujillo, Theory and Applications of Fractional Differential Equations, Elsevier, Amsterdam, 2006.

[11] M. Klimek, Stationarity-conservation laws for fractional differential equations with variable coefficients, J. Phys. A 35(31) (2002), 6675-6693.

[12] E. Lashkarian and S. Reza Hejazi, Group analysis of the time fractional generalized diffusion equation, Phys. A 479 (2017), 572-579.

[13] E. Lashkarian, S. Reza Hejazi and E. Dastranj, Conservation laws of $(3+\alpha)$-dimensional timefractional diffusion equation, Comput. Math. Appl. 75 (2018), 740-754.

[14] E. Lashkarian and S. Reza Hejazi, Exact solutions of the time fractional nonlinear Schrodinger equation with two different methods, Math. Methods Appl. Sci. (2018), DOI: 10.1002/mma.4770.

[15] S. Y. Lukashchuk, Conservation laws for time-fractional subdiffusion and diffusion-wave equations, Nonlinear Dynam. 80(1-2) (2015), 791-802.

[16] R. Magin, Fractional calculus models of complex dynamics in biological tissues, Comput. Math. Appl. 59 (2010), 1586-1593.

[17] F. C. Merala, T. J. Roystona and R. Magin, Fractional calculus in viscoelasticity, an experimental study, Commun. Nonlinear Sci. Numer. Simul. 15 (2010), 939-945.

[18] K. S. Miller and B. Ross, An Introduction to the Fractional Integrals and Derivatives-Theory and Applications, John Willey and Sons, New York, 1993.

[19] A. Naderifard, S. Reza Hejazi and E. Dastranj, Symmetry properties, conservation laws and exact solutions of time-fractional irrigation equation, Waves Random Complex Media, DOI: 10.1080/17455030.2017.1420943.

[20] E. Noether, Invariant variational problems, Transport Theory and Statistical Physics 1 (1971), 186-207.

[21] K. B. Oldham and F. Spanier, The Fractional Calculus, Academic Press, New York, 1974.

[22] P. Olver, Applications of Lie Groups to Differential Equations, Graduated Texts in Mathematics 107, Springer, New York, 1993.

[23] A. Ouhadan and E. H. Elkinani, Exact solutions of time fractional Kolmogorov equation by using Lie symmetry analysis, J. Fract. Calc. Appl. 1 (1974), 97-104.

[24] I. Podlubny, Fractional Differential Equations. An Introduction to Fractional Derivatives, Fractional Differential Equations, Some Methods of their Solution and Some of their Applications, Academic Press, San Diego, CA, 1999.

[25] S. Rashidi and S. Reza Hejazi, Symmetry properties, similarity reduction and exact solutions of fractional Boussinesq equation, Int. J. Geom. Methods Mod. Phys. 14(6) (2017), Article ID 1750083, 15 pages.

[26] S. Rashidi and S. Reza Hejazi, Analyzing Lie symmetry and constructing conservation laws for time-fractional Benny-Lin equation, Int. J. Geom. Methods Mod. Phys. 14(12) (2017), Article ID 1750170, 25 pages.

[27] S. Rashidi, S. Reza Hejazi and E. Dastranj, Approximate symmetry analysis of nonlinear Rayleigh-wave equation, Int. J. Geom. Methods Mod. Phys. 15(3) (2018), Artice ID 1850055, 18 pages.

[28] S. Samko, A. A. Kilbas and O. Marichev, Fractional Integrals and Derivatives: Theory and Applications, Gordon and Breach Science, Yverdon, Switzerland, 1993.

[29] E. Saberi and S. Reza Hejazi, Lie symmetry analysis, conservation laws and exact solutions of the time-fractional generalized Hirota-Satsuma coupled KdV system, Phys. A 492 (2018), 296-307.

[30] E. Saberi, S. Reza Hejazi and E. Dastranj, A new method for option pricing via time-fractional PDE, Asian-Eur. J. Math. 11(5) (2018), Article ID 1850074, 15 pages. 
[31] R. Sahadevan and T. Bakkyaraj, Invariant analysis of time fractional generalized Burgers and Korteweg-de Vries equations, J. Math. Anal. Appl. 2 (2012), 341-347.

[32] A. R. Seadawy, The solutions of the Boussinesq and generalized fifth-order KdV equations by using the direct algebraic method, Appl. Math. Sci. 12(82) (2012), 4081-4090.

[33] A. R. Seadawy, Stability analysis for Zakharov-Kuznetsov equation of weakly nonlinear ionacoustic waves in a plasma, Comput. Math. Appl. 67 (2014), 172-180.

[34] A. R. Seadawy, Fractional solitary wave solutions of the nonlinear higher-order extended KdV equation in a stratified shear flow: part I, Comput. Math. Appl. 70 (2015), 345-352.

[35] A. R. Seadawy, Dianchen Lu, bright and dark solitary wave soliton solutions for the generalized higher order nonlinear Schrodinger equation and its stability, Results in Physics 7 (2017), 43-48.

[36] A. R. Seadawy, Travelling-wave solutions of a weakly nonlinear two-dimensional higher-order Kadomtsev-Petviashvili dynamical equation for dispersive shallow-water waves, The European Physical Journal Plus 132(29) (2017), 13 pages.

[37] V. E. Tarasov, Fractional Dynamics: Applications of Fractional Calculus to Dynamics of Particles, Fields and Media, Springer-Verlag, Berlin-Heidelberg, 2010.

[38] V. E. Zakharov and E. A. Kuznetsov, On three-dimensional solitons, Journal of Experimental and Theoretical Physics 39 (1974), 285-286.

[39] G. Wanga and K. Fakhar, Lie symmetry analysis, nonlinear self-adjointness and conservation laws to an extended $(2+1)$-dimensional Zakharov-Kuznetsov-Burgers equation, Comput. \& Fluids 119 (2015), 143-148.

${ }^{1}$ Faculty of Mathematical Sciences, Shahrood University of Technology, Shahrood, SEMnAN, IrAN

Email address: a.naderifard1384@gmail.com

${ }^{2}$ Faculty of Mathematical Sciences, ShaHrood University OF TECHNOLOGY, Shahrood, SEMnAN, Iran

Email address: ra.hejazi@gmail.com

${ }^{3}$ Faculty of Mathematical Sciences, Shahrood University of Technology, Shahrood, SEmnan, Iran

Email address: dastranj.e@gmail.com 